\title{
TABAQUISMO EN COSTA RICA. IMPLICACIONES DEL FUMADO ACTIVO Y PASIVO EN LA POBLACION GENERAL ${ }^{1}$
}

\author{
SMOKING IN COSTA RICA. IMPLICATIONS OF ACTIVE AND \\ PASSIVE SMOKING IN THE GENERAL POPULATION
}

\author{
Marjorie Moreno Salas* \\ Julio Bejarano Orozco**
}

\begin{abstract}
RESUMEN
Este artículo presenta el resultado de un estudio que tuvo como objetivo, recopilar información sobre el consumo de tabaco, en una muestra nacional de 4645 encuestados, de 12 a 70 años, se exploró el nivel de exposición a la nicotina, tanto en fumadores activos como pasivos. Esta encuesta utilizó la técnica de entrevista en hogares y fue parte de un esfuerzo mayor, destinado a conocer la prevalencia y la incidencia del consumo de la nicotina y otras drogas. Mediante la determinación de los niveles de consumo en los informantes y en sus familiares, se estableció que la situación nacional en la materia es compleja y preocupante, dadas sus implicaciones en términos de salud pública, con base en los criterios de frecuencia, cantidad y patrones de uso.
\end{abstract}

PALABRAS CLAVE: TABACO * HÁBITO DE FUMAR * FUMADORES PASIVOS * FUMADORES ACTIVOS * SALUD PÚBLICA

\section{ABSTRACT}

This article presents the results of a study, which aimed to compile information on tobacco consumption in a national sample of 4645 subjects within 12 thru 70 years of age. Specific questions were designed to explore the level of exposition to nicotine, as much in active smokers as in passive ones. This survey used the technique of house-

1. Esta investigación fue financiada por el Instituto sobre Alcoholismo y Fármacodependencia.

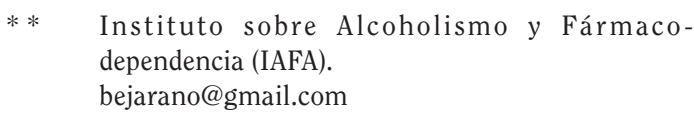

* Escuela de Psicología de la Universidad de Costa Rica y Escuela de Psicología, Universidad Católica de Costa Rica. maryyuri12@gmail.com 
hold interview and was part of a broader effort leading to know the prevalence and the incidence of consumption of other drugs. The determination of levels of consumption in the informants and their relatives was based on frequency criteria, amount and patterns of use. Outcomes suggested that situation at the local level is complex for its consequences on public health.

KEYWORDS: TOBACCO $*$ SMOKING * SMOKERS $*$ SECOND HAND SMOKING * PUBLIC HEALTH

\section{INTRODUCCIÓN}

Actualmente, es incuestionable que el consumo de tabaco genera perjuicios para la salud de quien lo consume, además que demanda de los países una importante cantidad de gastos en esta área. En el caso de Costa Rica, Castillo (2003), estimó los gastos de la Caja Costarricense del Seguro Social (CCSS) en unos us $\$ 25$ millones, los cuales representaron para el momento del estudio, el 55\% del costo total de atención de las enfermedades asociadas al consumo de tabaco. Dichos gastos correspondían únicamente, a la consulta externa $y$ al servicio de urgencias, las cuales fueron 858 mil consultas médicas generales y especializadas en todos los niveles de atención, dirigidas a pacientes con enfermedades relacionadas con el tabaquismo.

Otro aspecto de interés, es el carácter legal del tabaco. Dicho producto es accesible y pese a que según la Ley de Regulación del Fumado 7501, el consumo se encuentra prohibido para menores de edad, esto no los exonera de exponerse al humo de tabaco, el cual resulta doblemente dañino para la salud. Lo anterior se traduce en una mayor complejidad si se trabaja con otras poblaciones, como las mujeres embarazas, los adultos mayores, los niños/as o bien, empleados del área de servicios que regularmente se exponen a esta sustancia; tal es el caso de los meseros, animadores y otras personas, es decir, trabajadores de espacios socialmente destinados para el libre consumo de tabaco.

Lo anterior sugiere que, junto con la epidemia mundial del tabaquismo, se encuentra el tabaquismo pasivo o fumado pasivo, condición muy sensible, que al ser considerada un problema de salud pública, obliga a repensar los efectos sociales negativos del tabaco.
En Costa Rica, existe información diversa sobre el tabaco y el tabaquismo, la cual corresponde a diferentes poblaciones. La mayor cantidad de aportes en este sentido los ha realizado el Instituto sobre Alcoholismo y Farmacodependencia (IAFA). En esta línea, figuran investigaciones con la población general (Bejarano, Fonseca y Sánchez, 2009; Sandí y Molina, 2008; Bejarano y Ugalde, 2003) y jóvenes (Bejarano, 2004-2005). Sin embargo, en Costa Rica se desconocen cifras de interés sobre la cantidad de fumadores pasivos, la edad de los mismos y los principales espacios en que se ven expuestos a la corriente secundaria, entre otros datos.

Ahora bien, según los datos del estudio más reciente del IAFA (Bejarano, Fonseca y Sánchez, 2009) la prevalencia de vida de fumado en el 2006, fue de 31,5\%, mientras que, para el 2001, los datos de prevalencia se establecieron en $29,9 \%$. La distribución por sexo de la prevalencia de vida durante el 2006 para los hombres fue de $41 \%$, mientras que para las mujeres fue de $21,5 \%$. Si se revisa la prevalencia de consumo durante el último año, la diferencia entre los hombres $y$ las mujeres es igualmente amplia, al ser la primera un $23,2 \%$, en tanto la femenina es $9,4 \%$.

La incidencia del tabaquismo es de 24 por 1000 habitantes, esto equivale a afirmar que, durante el año anterior a la encuesta, 24 personas de cada 1000 se iniciaron en el consumo. La edad promedio de inicio en los hombres que han fumado, fue de 15,84 años, la cual es significativamente inferior que la de las mujeres, la cual fue de 17,07 años.

Sin embargo, aunque estos datos permiten escudriñar los patrones de consumo de los/as costarricenses, no dan cuenta del fumado pasivo, 
entendido como inhalar el humo exhalado por los/as fumadores. Se trata de un tema de interés nacional y de salud pública, pues una presunción sería que muchos de los padres/madres de familia podrían estar consumiendo tabaco en sus hogares, cerca de otros familiares $y$ por lo tanto, estarían perjudicando las condiciones de salud de más de una persona.

Sin embargo, la situación trasciende este hecho: en las sociedades latinoamericanas y de otros continentes, el tabaco es una droga de consumo social, asociada con la búsqueda de estados de relajación y vinculada con el consumo de otras drogas como el café y el alcohol, en espacios socialmente destinados para eso, tal es el caso de los bares, restaurantes, cafés $y$ salones de baile, entre otros, lo cual implica la exposición a grandes cantidades de humo de tabaco. Es decir, no solo los hogares son espacios potenciales para fumadores pasivos, sino también los diversos espacios públicos; generalmente, lugares cerrados en los que se permanece cierto número de horas seguidas. Los retos para conocer los niveles del fumado pasivo transcienden el ámbito familiar y se acercan al espacio comercial y de servicios, especialmente los del entretenimiento.

Por otra parte, también destacan las campañas antitabaco, las cuales son esfuerzos institucionales por incidir en los patrones de consumo, ya sea para eliminarlos o reducirlos, aumentar la edad de inicio o controlar el impacto del consumo; sin embargo, generalmente, estos esfuerzos son de prevención universal, por ello los mensajes suelen ser muy amplios y dirigidos a la población general.

En Costa Rica, resaltan esfuerzos considerados más bien dispersos y poco articulados. Al respecto, se desconoce el impacto de las campañas realizadas, las cuales han sido evaluadas globalmente. Un ejemplo es la valoración del programa "Trazando el camino" (Bejarano, Ugalde y Morales, 2005), del cual se conocen los cambios en el consumo de tabaco, pero se desconoce el impacto del consumo de tabaco individual en terceros.

El foco del presente estudio fue trabajar sobre el fumado activo y pasivo, ahondando en datos empíricos al respecto. El fumado pasivo consiste en que personas no fumadoras se vean expuestas al humo de tabaco exhalado por fumadores/as (corriente secundaria) (Carrión y Hernández, 2002), junto con esto, se resalta que el tabaco está compuesto aproximadamente por 5000 sustancias tóxicas, asociando al fumado pasivo con cáncer de pulmón, enfermedades cardiovasculares, asma bronquial y síntomas respiratorios, entre otros (Carrión y Hernández, 2002).

En Costa Rica, una tercera parte de los $y$ las adolescentes encuestados vivían con personas fumadoras. Uno de cada cinco individuos indicó haber estado en la presencia de un fumador en el hogar, en tres de los siete días previos a la recolección de datos, mientras un tercio de los y las participantes aseguró haber estado expuesto al humo de tabaco en lugares diferentes a la casa de habitación (Bejarano, 2001).

El hábito de consumir tabaco suele instaurarse durante la adolescencia, de ahí la relevancia de esta población. La encuesta mundial sobre tabaquismo en jóvenes, señala que durante el 2008, en Costa Rica, la prevalencia del fumado activo en personas de 13 a 15 años era cercana al 10\%. Además, señala que la mayoría de los/as jóvenes de Centroamérica y Suramérica respiran el humo de los demás. Cabe mencionar que este nivel de prevalencia cayó de $18 \%$ en 1999 , a un $10 \%$ en el 2008 (Bejarano, 2009).

En la mitad de las zonas participantes del estudio, más del 50\% de los jóvenes se ven expuestos al humo de los demás, tanto en lugares públicos como en la casa de habitación, lo cual no debe pasar inadvertido, especialmente, si se revisa en función de variables como: la calidad de vida, las condiciones de salud física $y$ emocional de los niños/as y adolescentes, además de elementos económicos, entre otros.

Otro tema de interés, son las opciones para la desestimulación del consumo activo y pasivo. Datos de investigación de la Organización Panamericana de la Salud y la Organización Mundial de la Salud (OPS/OMS, 2002) indican que en el Área Andina, existe la mayor proporción de jóvenes (90\% en Trujillo, Perú) que prohibirían el consumo de tabaco en lugares públicos, seguido por Costa Rica. 
Destaca que salvo en 2 de las 30 regiones restantes, más del $70 \%$ de los jóvenes encuestados prohibiría fumar en lugares públicos. En alguna medida, esto se asocia con la tolerancia social y posiblemente, con la toma de consciencia sobre la problemática del consumo.

El punto anterior es fundamental, pues podría indicar la presencia de futuros escenarios favorables, en tanto no fumadores/as estén más dispuestas a exigir sus derechos o bien, mucho más tendientes a exigir y aplicar asuntos normados, por ejemplo, cuándo, dónde y bajo qué condiciones deberían las personas consumir tabaco.

En este sentido, no debe perderse de vista que los fumadores pasivos se ven afectados directamente por los hábitos de consumo de los fumadores activos, pues se asocia con el lugar de consumo y cantidad de cigarros consumidos, ventilación, entre otros (Carrión y Hernández, 2002).

Un estudio realizado en hospitales, escuelas, edificios públicos, restaurantes y aeropuertos de las capitales de Argentina, Brasil, Chile, Costa Rica, Paraguay, Perú y Uruguay, mediante la instalación de censores, demostró concentraciones de nicotina (muchas de ellas altas), en la gran mayoría de los sitios anteriores (Navas et ál., 2004).

Se ha estimado que el $80 \%$ de los no fumadores viven con algún fumador $y$ se ven expuestos al humo de tabaco de corriente secundaria o lateral; o bien, se exponen a esta sustancia en el lugar de trabajo. Es relevante que pese a la posibilidad de eliminar el consumo pasivo por medio de prácticas, como fumar en espacios abiertos o no fumar en presencia de otras personas, este se mantiene (Carrión y Hernández, 2002).

Más alarmante aún, es el "síndrome de tabaco fetal", que implica bajo peso al nacer, alteraciones endocrinas y alteraciones en la función pulmonar, entre otros riesgos. Esta condición de salud constituiría una violación de los derechos de los niños/as, pues un neonato enfrentaría problemas de salud, prevenibles $y$ asociados con negligencia por parte de los padres/madres (Carrión y Pellicer, 2002).

Se ha estimado que la exposición de los no fumadores al humo de los fumadores, causa entre 1100 y 7800 muertes al año, en Canadá (Ontario Tobacco Research Unit, University of Toronto, 2001). Esta cifra dió pie a promulgar la eliminación de toda exposición involuntaria al humo del tabaco, pues los ambientes mejor ventilados no constituyen necesariamente una solución al problema, aún con la tecnología más sofisticada. Desde el decenio anterior, se ha informado que por cada ocho fumadores muertos, fallece un no fumador (Taylor, Johnson y Kazemi, 1992).

Finalmente, el tema es complejo y posee múltiples aristas en función de las poblaciones afectadas y las posibles medidas de control. De especial relevancia, es el esfuerzo de la Organización Mundial de la Salud (OMS), con la promulgación del Convenio Marco para el control del tabaco, mismo que en Costa Rica no ha entrado en vigencia, pero ha tenido cierta difusión. Desde el Convenio se han propuesto elementos relevantes en varios aspectos como el económico, el social y el comercial.

Debido a la escasa información sobre el tema, el propósito del presente estudio es aportar datos empíricos asociados con el tabaquismo activo y especialmente el pasivo.

\section{MATERIALES Y MÉTODO}

\section{PARTICIPANTES}

Los datos del presente estudio corresponden a un diseño transversal y los detalles metodológicos son los propios de la encuesta de hogares, llevada a cabo por el IAFA, en el 2001. Su población meta estuvo conformada por todos los habitantes de Costa Rica, costarricenses o no, con edades entre los 12 y 70 años, residentes en una vivienda que tenían al menos un año de habitarla. Para determinar el tamaño de la muestra, se utilizaron las estimaciones de población para el año 2000 , realizadas por el Instituto Nacional de Estadística $y$ Censos (INEC); la misma fue establecida en 4645 individuos (2350 hombres y 2296 mujeres). La asignación del número de individuos fue proporcional al tamaño de cada provincia, al grupo de edades correspondientes y a la distribución por sexo. 
La utilización de los datos de la encuesta de 2001, existiendo una encuesta nacional más reciente, obedeció a que en aquella se inquirió información específica sobre el fumado pasivo.

\section{INSTRUMENTO}

El cuestionario utilizado para recolectar la información fue el empleado durante las rondas de investigación del IAFA, iniciadas en 1990. El instrumento contó con algunas variaciones para aumentar la cantidad de información recopilada y se agregaron una serie de preguntas para medir el consumo activo y pasivo.

\section{PROCEDIMIENTO}

El cuestionario fue aplicado en las casas de habitación de los informantes, por entrevistadores/as entrenados, con el fin de motivar a los entrevistados para contestar sinceramente cada una de las preguntas. Los casos fueron seleccionados al azar, como parte de una muestra probabilística en tres etapas y representativa de la población nacional. Previo a la aplicación del cuestionario, cada informante brindó su consentimiento verbal, participando voluntariamente en el estudio.

Los datos derivados de los cuestionarios fueron sistematizados utilizando el Paquete Estadístico para Ciencias Sociales (SPSS) para digitación. Posteriormente, se realizaron análisis estadísticos descriptivos, así como, análisis de frecuencias y análisis de contingencias. Seguidamente, se detallan los resultados del presente estudio.

\section{RESULTADOS}

\section{DESCRIPCIÓN DE LA MUESTRA}

Un 50,5\% de la muestra del estudio estuvo constituida por hombres. Tal y como se mencionó previamente, dicha muestra es representativa de la población nacional y se seleccionó por cuotas, según provincia y edad.

En el siguiente cuadro se recopilaron las principales variables sociodemográficas que se tomaron en cuenta en el estudio.
TABLA 1

DISTRIBUCIÓN DE LOS RESULTADOS DE LAS VARIABLES SOCIODEMOGRÁFICAS

\begin{tabular}{|c|c|c|}
\hline & RANGOS/CATEGORÍAS & $\%$ \\
\hline \multirow[t]{12}{*}{ 1.Edad } & $12-14$ & 9,4 \\
\hline & $15-19$ & 18,1 \\
\hline & $20-24$ & 12,3 \\
\hline & $25-29$ & 11,1 \\
\hline & $30-34$ & 10,8 \\
\hline & $35-39$ & 10,5 \\
\hline & $40-44$ & 7,3 \\
\hline & $45-49$ & 6,5 \\
\hline & $50-54$ & 4,3 \\
\hline & $55-59$ & 4 \\
\hline & $60-64$ & 2,3 \\
\hline & $65-70$ & 3,3 \\
\hline \multirow[t]{6}{*}{ 2.Estado civil } & Soltero & 45,1 \\
\hline & Casado & 39,3 \\
\hline & Viudo & 1,8 \\
\hline & Divorciado & 2,4 \\
\hline & Unión libre & 10,2 \\
\hline & Separado & 1,2 \\
\hline \multirow[t]{8}{*}{ 3.Ocupación } & Sector primario & 7,7 \\
\hline & Sector secundario & 17,5 \\
\hline & Sector terciario & 15,1 \\
\hline & Estudiante & 21,3 \\
\hline & Ama de casa & 27,6 \\
\hline & Jubilado & 3,4 \\
\hline & No tiene trabajo & 6,6 \\
\hline & Otro & 0,8 \\
\hline \multirow[t]{5}{*}{ 4.Religión } & Católico practicante & 62,5 \\
\hline & Católico no practicante & 11,7 \\
\hline & Protestante & 16,5 \\
\hline & No tiene & 6,7 \\
\hline & Otra & 2,6 \\
\hline
\end{tabular}

Fuente: Elaboración propia. $\mathrm{n}=4645$

Como se observa en el tabla anterior, la edad que predomina en el grupo de personas encuestadas se encuentra en el rango de los 15-19 años, seguido por el de 20-24 años, mientras que el menor porcentaje de participantes corresponde a los adultos mayores con un 3,3\%.

En cuanto al estado civil, el mayor porcentaje de personas corresponde a individuos que se encuentran solteros y casados $(45,1 \%$ y 39,3\%, respectivamente). Las personas en unión libre se agrupan en un 10,2\%. Respecto a la ocupación, los puestos de trabajo de los y las 
participantes se concentran en ser estudiante con un $21,3 \%$ y ama de casa con un $27,6 \%$, seguido por personas que se desempeñan en el sector secundario de la economía con $17,5 \%$ y un $15,1 \%$ en el terciario. La escolaridad, se concentró principalmente, en primaria completa con un $24 \%$, seguido de la secundaria incompleta, con 30,6\% (ver la tabla 2 ).

TABLA 2

DISTRIBUCIÓN DE LOS RESULTADOS DE LAS VARIABLES SOCIO-DEMOGRÁFICAS

\begin{tabular}{llc}
\hline & CATEGORÍA & $\%$ \\
\hline 1.Provincia & San José & 35,1 \\
& Alajuela & 17,9 \\
& Cartago & 11,2 \\
& Heredia & 9,1 \\
& Guanacaste & 7,7 \\
& Puntarenas & 9,9 \\
& Limón & 9,1 \\
\hline 2.Escolaridad & Primaria incompleta & 18,1 \\
& Primaria completa & 24,0 \\
& Secundaria incompleta & 30,6 \\
& Secundaria completa & 10,8 \\
& Universitaria incompleta & 7,4 \\
& Universitaria completa & 6,6 \\
& Para-universitaria & 1,0 \\
& Sin educación formal & 1,3 \\
& Otra & 0,2 \\
\hline 3.Ingresos familiares & Nivel bajo & 21,6 \\
& Nivel medio-bajo & 50,4 \\
& Nivel medio & 24 \\
& Nivel alto & 4 \\
\hline
\end{tabular}

Fuente: Elaboración propia. $\mathrm{n}=4645$

Por último, los ingresos familiares se dividieron en cuatro categorías, donde el mayor porcentaje corresponde a un nivel medio-bajo (50,4\%). La proporción de personas con bajos ingresos $(21,6 \%)$ guarda relación con los datos de otras fuentes sobre la pobreza en el país (Proyecto Estado de la Nación, 2001).

\section{CONSUMO DE TABACO}

Del total de participantes, un 30\% $(n=1374)$, aseguró haber probado el tabaco alguna vez en la vida. En estos, el promedio de edad de iniciación fue de 16,30 años (la moda 15 años) y el promedio de años de fumado 12,77.

TABLA 3

FUMADORES DE ALGUNA VEZ SEGÚN ÚLTIMA OCASIÓN DE FUMADO

\begin{tabular}{lcc}
\hline & $f$ & $\%$ \\
\hline En los últimos 30 días & 723 & 52,6 \\
Más de un mes, pero menos de un año & 68 & 4,9 \\
Hace más de un año & 582 & 42,3 \\
\hline TOTAL & 1373 & 100 \\
\hline
\end{tabular}

Fuente: Elaboración propia.

De acuerdo con los datos presentados en la tabla 3 , cabe destacar la presencia de una tasa de ex fumado nada despreciable $(42,3 \%)$, pero superada por los fumadores acti$\operatorname{vos}(52,6 \%)$.

El promedio de días que los y las participantes fumaron fue de 24 , lo cual sugiere un predominio de fumado casi diario (la moda es 30 días). El promedio de cigarrillos fumados en el último mes corresponde a 12,6. Aproximadamente, a la mitad de esta población le hace falta el tabaco $(46 \%, n=328)$, si no consume la cantidad acostumbrada.

Un aspecto relevante que se hace notar en los resultados, corresponde a una posible recomendación médica, acerca de dejar de fumar. Así un 40,1\% (n=314) aseguró que ha recibido dicha recomendación de un profesional de la salud, mientras que el 59,9\% ( $\mathrm{n}=470)$ no la ha recibido.

Un dato interesante es que un $66,5 \%$ $(n=522)$ de los fumadores ha intentado dejar de fumar, lo cual revela la importancia que tiene la cesación para la mayoría, no obstante, esta intención no siempre culmina de manera exitosa. Las personas que han intentado suspender el consumo, han realizado entre uno y dos intentos en el último año, seguidos por las personas que han realizado de tres a cuatro tentativas (tabla 4). 
TABLA 4

INTENTOS POR DEJAR DE FUMAR EN EL ÚLTIMO AÑO

\begin{tabular}{lcc}
\hline & $f$ & $\%$ \\
\hline 1 a 2 veces & 323 & 64 \\
3 a 4 veces & 118 & 23,4 \\
5 a 6 veces & 33 & 6,5 \\
7 y más & 31 & 6,1 \\
\hline TOTAL & 505 & 100 \\
\hline
\end{tabular}

Fuente: Elaboración propia.
Ahora bien, las principales razones que motivaron a las personas a dejar de fumar, fueron: "es malo para la salud", con un $72,2 \%$, seguido por "no quisiera defraudar a mi familia", con un $8,1 \%$. En total, se contabilizaron ocho razones diferentes que merecen consideraciones más detalladas.

TABLA 5

RAZONES PARA DEJAR DE FUMAR ENTRE LOS EX FUMADORES

\begin{tabular}{lcc}
\hline & $f$ & $\%$ \\
\hline 1. Es malo para la salud & 374 & 72,2 \\
2. De acuerdo a mis principios es malo & 11 & 2,1 \\
3. No quisiera defraudar a mi familia & 42 & 8,1 \\
4. Mi iglesia está en contra de eso & 3 & 0,6 \\
5. No tengo edad para hacerlo & 1 & 0,2 \\
6. Me perjudica & 30 & 5,8 \\
7. Cuesta mucho dinero & 25 & 4,8 \\
8. Otro & 32 & 6,2 \\
\hline TOTAL & 518 & 100 \\
\hline
\end{tabular}

Fuente: Elaboración propia.

Las personas no fumadoras o que han dejado de fumar alegan que se mantienen en esa posición pues "es malo para la salud" (69,7\%). En segundo lugar, están quienes indican "de acuerdo con mis principios es malo" $(14,2 \%)$.

Cabe notar que en este caso al igual que el anterior, la primera opción es la mayormente señalada como un motivo para haber dejado de fumar o no haberlo hecho hasta el momento (ver tablas 5 y 6 ).

De igual forma, por cada vivienda se registró el número de personas que fumaron durante el último mes. En la mayoría de los casos, solamente una persona fumaba por vivienda, lo cual corresponde a un 73,6\% de los/ as participantes, a esta cifra le sigue un 19,9\% de viviendas en las que dos personas fumaban.
TABLA 6

RAZONES PARA NO VOLVER A FUMAR O NO HABER FUMADO NUNCA

\begin{tabular}{lll}
\hline & $f$ & $\%$ \\
\hline 1.Es malo para la salud & 2615 & 69,7 \\
2.De acuerdo a mis principios es malo & 532 & 14,2 \\
3.No quisiera defraudar a mi familia & 118 & 3,1 \\
4.Mi iglesia está en contra de eso & 46 & 1,2 \\
5.No tengo edad para hacerlo & 12 & 0,3 \\
6.Me perjudica & 161 & 4,3 \\
7.Cuesta mucho dinero & 22 & 0,6 \\
8.Otro & 244 & 6,5 \\
\hline TOTAL & 3750 & 100 \\
\hline
\end{tabular}

Fuente: Elaboración propia. 
TABLA 7

CANTIDAD DE FUMADORES POR VIVIENDA EN VIVIENDAS CON AL MENOS UN FUMADOR

\begin{tabular}{ccc}
\hline CANTIDAD & $f$ & $\%$ \\
\hline 1 & 1154 & 73,6 \\
2 & 313 & 19,9 \\
3 & 76 & 4,8 \\
4 & 16 & 1,0 \\
5 & 5 & 0,3 \\
6 & 2 & 0,1 \\
7 & 2 & 0,1 \\
10 & 1 & 0,1 \\
\hline TOTAL & 1569 & 100 \\
\hline
\end{tabular}

Nota: 1569 corresponde a un 34\% de las viviendas.

Fuente: Elaboración propia.

Otros valores que se aprecian en la tabla 7 , si bien se trata de relativamente pocos casos, el impacto sobre la salud de los demás integran- tes de la vivienda se interpreta como negativo.

Seguidamente, la cantidad de informantes (1569) que habitan al menos con un fumador y fumaron durante el último mes, se asocia con la condición del informante como fumador en el último año $(p<0,001)$.

Entre los no fumadores es mayor la proporción de quienes mencionan que nadie fumó en su vivienda durante el último mes (ver tabla 8). Mientras, entre los fumadores, es mayor la presencia en su vivienda de alguien más que haya fumado en el último mes.

En cuanto a los fumadores activos que residen en los hogares de los informantes, predominan sus padres $y$ hermanos (ver tabla 9). Hay una proporción significativamente mayor de padres, madres, hermanos, cónyuges y otros miembros fumadores en el hogar de los informantes fumadores que en los hogares de los informantes no fumadores. No hay diferencia significativa respecto a la presencia de abuelos o tíos fumadores. Las afirmaciones anteriores son estadísticamente significativas $(p=0,05)$.

TABLA 8

CANTIDAD DE FAMILIARES QUE FUMAN

SEGÚN FUMADORES Y NO FUMADORES POR VIVIENDA

\begin{tabular}{ccc}
\hline NRO. DE FAMILIARES QUE FUMAN & $\begin{array}{c}\text { \% DE NO FUMADORES } \\
(\mathrm{n}: 705)\end{array}$ & $\begin{array}{c}\text { \% DE FUMADORES } \\
(\mathrm{n}: 864)\end{array}$ \\
\hline 1 & 65,3 & 80,3 \\
2 & 24,8 & 16,0 \\
3 & 7,9 & 2,3 \\
4 & 0,9 & 1,2 \\
5 & 0,6 & 0,1 \\
6 & 0,1 & 0,1 \\
7 & 0,3 & 0,0 \\
10 & 0,1 & 0,0 \\
\hline TOTAL & 100 & 100 \\
\hline
\end{tabular}

Fuente: Elaboración propia 
TABLA 9

DISTRIBUCIÓN DE LOS FAMILIARES FUMADORES

SEGÚN INFORMES DE FUMADORES Y DE NO FUMADORES

\begin{tabular}{lcccc}
\hline $\begin{array}{l}\text { PRESENCIA EN EL HOGAR DE } \\
\text { OTROS FUMADORES }\end{array}$ & $\begin{array}{c}\text { EN HOGARES DE } \\
\text { INFORMANTES N } \\
\text { FUMADORES }\end{array}$ & $\begin{array}{c}\text { EN HOGARES } \\
\text { DE INFORMANTES } \\
\text { FUMADORES }\end{array}$ & TOTAL \\
\hline Padre & $6,66 \%$ & $9,72 \%$ & $7,19 \%$ \\
Madre & $2,95 \%$ & $5,68 \%$ & $3,42 \%$ \\
Cónyuge & $4,85 \%$ & $6,94 \%$ & $5,21 \%$ \\
\hline Hermanos (cantidad) & 1 & $4,98 \%$ & $10,73 \%$ & $5,97 \%$ \\
& 2 & $1,03 \%$ & $3,41 \%$ & $1,44 \%$ \\
& 3 & $0,18 \%$ & $0,38 \%$ & $0,22 \%$ \\
\hline Abuelos & & $0,50 \%$ & $0,38 \%$ & $0,48 \%$ \\
\hline Tíos (cantidad) & 1 & $0,76 \%$ & $1,26 \%$ & $0,85 \%$ \\
& 2 & $0,11 \%$ & $0,00 \%$ & $0,09 \%$ \\
& 3 & $0,00 \%$ & $0,13 \%$ & $0,02 \%$ \\
\hline Otras personas (cantidad) & 1 & $3,29 \%$ & $4,67 \%$ & $3,53 \%$ \\
& 2 & $0,34 \%$ & $1,26 \%$ & $0,50 \%$ \\
& 3 & $0,11 \%$ & $0,13 \%$ & $0,11 \%$ \\
& 4 & $0,05 \%$ & $0,00 \%$ & $0,04 \%$ \\
\hline
\end{tabular}

Fuente: Elaboración propia.

\section{DISCUSIÓN Y CONCLUSIONES}

Los datos analizados en este estudio ponen de relieve la importancia de la exposición al humo de terceros, tanto por parte de sujetos no fumadores como de los fumadores mismos; junto al hecho que una tercera parte de los informantes refirieron que al menos una persona en su casa fumaba. Además, se tiene que en la población escolarizada, con edades entre $13 y$ 15 años, un porcentaje mayor de la mitad indicó haber estado expuesto al humo de terceros en lugares públicos (Bejarano, 2004-2005).

A lo anterior, se agrega la inexistencia de un nivel seguro para la exposición al humo de otros, pues por más breve que sea la exposición, esta puede provocar cambios en las vías respiratorias superiores de personas sanas, además, de producir ataques más frecuentes de asma en los niños con este padecimiento (US. Department of Health and Human Services, 2006).

Los no fumadores que conviven con fumadores, tienen un riesgo 35 veces mayor de contraer cáncer de pulmón que aquellos que no conviven con fumadores, pues es de conocimiento que con solo 30 minutos de exposición al humo ajeno, este puede causar daño al corazón de manera semejante al que se causaría en un fumador habitual. A juzgar por la experiencia de algunos países desarrollados, es sabido que la cesación ayuda a evitar el exceso de riesgos derivados del tabaquismo. En países de ingresos medios o bajos la cesación parece ser un fenómeno menos generalizado que en los primeros.

A continuación, se presentan algunas consideraciones sobre la importancia de los resultados del presente estudio, en tanto, la condición de fumadores $y$ no fumadores expone a las personas a importantes riesgos de salud.

Las primeras evidencias acerca de la sensibilidad de los no fumadores hacia los componentes del tabaco, provienen de estudios que demostraron que una exposición tan breve como 30 minutos al humo de un fumador, era capaz de activar las plaquetas hasta niveles cercanos a los que exhibían sujetos fumadores; de manera tal, que el fumado pasivo aumenta la morbilidad de las células endoteliales en la sangre (Glantz y Parmley, 2001). Estos efectos inmediatos en las plaquetas, posiblemente actúan sinérgicamente con los efectos sobre 
la función endotelial y dan fuerza a la idea de que la curva de dosis/respuesta para efectos cardiovasculares, asociada a la exposición al humo, no es lineal, pero muestra un efecto sustancial a dosis relativamente bajas, si se compara con un fumador activo. Los modelos animales han demostrado que la exposición a un cigarrillo por día, induce a cambios ateroescleróticos. Un hecho interesante, es que el fumado pasivo no provoca efectos adicionales en el fumador activo, lo cual sugiere que los procesos bioquímicos y celulares subyacentes, se saturan en las dosis que los fumadores pasivos experimentan (Otsuka et ál., 2001). De acuerdo con García, Sáenz, García y Grau (2007), los fumadores pasivos parecen tener mayor riesgo de ictus isquémico que los no fumadores no expuestos al humo del tabaco. Este riesgo, no resultó muy distinto del alcanzado por los fumadores activos; los resultados apoyan lo hallado en estudios previos y refuerzan el vínculo entre ser un fumador pasivo $y$ desarrollar ictus isquémico.

Adicionalmente, es pertinente mencionar que estos riesgos se asocian directamente con todas las personas que puedan estar expuestas al humo de tabaco. En este caso, puede ser cualquier persona, desde niños/as hasta adultos mayores $y$ mujeres en estado de embarazo. Es pertinente, por tanto, considerar lo anterior en el diseño de las políticas sanitarias adelantadas, en parte con el Convenio Marco para el Control del Tabaco de la OMS. Sin embargo, sigue siendo necesario contribuir socialmente con la disminución de los riesgos de salud asociados, pues se puede argumentar que, posiblemente, muchas personas no contemplen los beneficios de cesar el fumado o bien, evitar exponerse al humo.

Al respecto, es conveniente señalar que los esfuerzos en salud deben enfocarse por un lado, en prevenir el fumado y por el otro, evitar $y$ disminuir el fumado pasivo. Además, medidas tendientes a que los fumadores busquen espacios abiertos para el consumo de tabaco, aprobar políticas sanitarias sostenidas y ajustadas a poblaciones más vulnerables, tal es el caso de los meseros y personas que trabajan en bares, entre otros.
Los datos del presente estudio pueden contribuir indirectamente con el diseño de campañas, pues se cuenta con información sobre aspectos motivacionales asociados con abstenerse del consumo. También, los informantes manifestaron los diversos intentos realizados para dejar de fumar, sin haberlo conseguido, asimismo, señalaron que han recibido indicaciones médicas para dejar de fumar.

Otro de los temas relevantes, es el inicio del consumo de tabaco y la posterior instauración de la dependencia nicotínica; el final de la infancia e inicio de la adolescencia es la etapa de desarrollo característica de esta condición. Para prevenir el consumo de tabaco a nivel individual, además de trabajar de forma holística con esta población, se debe integrar el abordaje familiar, pues tal y como lo señalan los datos anteriores, el consumo de tabaco y fumado pasivo son fenómenos presentes en el contexto de la familia en el hogar.

Finalmente, en el nivel investigativo conviene lograr nuevos estudios sobre fumado activo y pasivo que refieran diferentes grupos poblacionales, así como, evaluar el impacto de las medidas que oportunamente se implementen en Costa Rica, luego de la puesta en ejecución del Convenio Marco para el Control del Tabaco.

\section{REFERENCIAS}

Bejarano, J. "El consumo de tabaco en la población joven costarricense: hallazgos de la encuesta mundial sobre tabaco en jóvenes". Revista Costarricense de Salud Pública 10. 2001: 18-19. [Revista digital] En: <http: //www.scielo.sa.cr.> [consultado el 15 de enero de 2008].

Bejarano, J. "El consumo de tabaco en jóvenes costarricenses escolarizados. Comparación de los resultados obtenidos en la Encuesta Mundial sobre Tabaquismo en jóvenes (GYTS) en 1999 $y$ el 2002". Revista costarricense de psicología 36-37. 2004-2005: 11-21.

Bejarano, J. "Changes over time in Costa Rican data. The three rounds of the GYTS project". Conferencia presentada en la celebración del 10 aniversario 
del Sistema Mundial de Vigilancia del Tabaco. Atlanta, junio 2009: 15-19.

Bejarano, J.; Fonseca, S. y Sánchez, G. Consumo de drogas en Costa Rica. Resultados de la Encuesta Nacional 2006. San José, Costa Rica: Instituto sobre Alcoholismo y Farmacodependencia, 2009.

Bejarano, J. y Ugalde, F. Consumo de drogas en Costa Rica. Resultados de la encuesta nacional del 2000-2001. San José, Costa Rica: Instituto de Alcoholismo y Farmacodependencia, 2003.

Bejarano, J.; Ugalde, F. y Morales, D. "Evaluación de un programa escolar en Costa Rica basado en habilidades para vivir". Revista Adicciones 17. 2005: 71-80.

Carrión, F. y Hernández, J. R. "El tabaquismo pasivo en adultos". Archivos de Bronconeumonología 38 (3). 2002: 137-146.

Carrión, F. y Pellicer, C. "El tabaquismo pasivo en la infancia. Nuevas evidencias". Prevención del tabaquismo 4 (1). 2002: 20-25.

Castillo, J. Estimación del costo de atención en el Seguro de Salud de la Caja Costarricense de Seguro Social atribuible al consumo de tabaco. Caja Costarricense de Seguro Social. Departamento de Estadística, 2003.

García, C. et ál. "El fumador pasivo como factor de riesgo cerebro vascular". Revista de Neurología 45 (10). 2007: 577-581.

Glantz, S. y Parmley, W. "Even a little secondhand smoke is dangerous". Journal of the American Medical Association 286 (4) (reprinted). 2001: 462-463.

Navas, A. et ál. "Second hand tobacco smoke in public places in Latin America, 20022003". Journal of the American Medical Association 291 (22). 2004: 2741-2745.

Ontario Tobacco Research Unit, University of Toronto. "Protection from second-hand tobacco smoke in Ontario. A review of the evidence regarding best practices". [Report of the Ontario Tobacco Research Unit]. Toronto, Ontario. University of Toronto, 2001.

OPS/OMS. "Encuesta mundial sobre tabaquismo en la juventud: resultados en las Américas”. Boletín Epidemiológico 23. 2002: 1-2.

Otsuka, R. et ál. "Acute effects of passive smoking on the coronary circulation in healthy young adults". Journal of the American Medical Association 286 (4). 2001: 436-441. En: <http:// www.ncbi.nlm.nih.gov/entrez/query/. fcgi? cmd=Retrieve \&db=PubMed\&li st_uids $=11466122 \& d o p t=$ Abstract $>$ [consultado el 21 de mayo de 2009].

Proyecto Estado de la Nación. Estado de la Nación en Desarrollo Humano Sostenible: Sétimo Informe. 2000. San José, Costa Rica, 2001.

Sandí, L. y Molina, D. Guía de intervención para el tratamiento de la dependencia al tabaco. San José: IAFA, 2008.

Taylor, A.; Johnson, D. y Kazemi, H. "Environmental tobacco smoke and cardiovascular disease. A position paper from the council on cardiopulmonary and critical care, American Heart Association”. Circulation 86. 1992: 699702.

us Department of Health and Human Services. The health consequences of involuntary exposure to tobacco smoke: a report of the surgeon general. us. Department of Health and Human Services, Centers for Disease Control and Prevention, National Center for Chronic Disease Prevention and Health Promotion, Office on Smoking and Health, 2006.

Fecha de ingreso: 23/06/2011 Fecha de aprobación: 13/09/2011 
